\title{
Impact Analysis of Mobility Models on Routing Strategies for Delay Tolerant Networks
}

\author{
Sanjay Kumar ${ }^{1}$, Sudhakar Pandey ${ }^{2}$ \\ Department Of Information Technology, NIT Raipur,492010,India \\ ${ }^{1}$ skumar.it@nitrr.ac.in² spandey.it@nitrr.ac.in
}

\begin{abstract}
Delay Tolerant Networks (DTNs) has received a lot of attention by researchers in the recent years. It has been applied in many areas where end to end path connectivity does not exist. This technology uses the mobility of nodes and hence mobility models are considered very essential while evaluating any routing protocols in DTNs. Selection of an appropriate model is important for evaluation for performance of the protocol and checking its applicability in real world. We provide a detailed study of mobility models used in DTNs and perform comparative analysis of their effect on routing protocols of DTNs. We have used criterion such as transmission range, bundle time to live and number of nodes in our simulation. In our experiments, shortest path map based mobility model (SPMBM) performs better with the routing protocols used in delay tolerant network selected for our simulation.
\end{abstract}

Keywords: Delay Tolerant Network, Mobility Models, Routing protocols.

\section{INTRODUCTION}

Delay Tolerant Networks [12] can be deployed in the environment where end to end connectivity does not exist for most of the time. DTNs use store carry and forward paradigm to deliver the messages from source to destination. Nodes in DTN are assumed to be capable of storing, carrying and forwarding messages received from other nodes. It is very difficult to test the performance of routing protocols in real environment; hence most routing protocols are evaluated on simulators based on different mobility model. Mobility models and their characteristics have been studied by number of researchers and are updated accordingly to achieve realism.

\section{A. Our Contribution}

In this paper, we study theimpact of mobility modelson the performance of various routing protocols of delay tolerant networks. For this purpose, we developed suitable environment for each routing protocol using ONE simulator and studied the effect of different mobility models on performance metrics like delivery probability, overhead ratio, average latency and average buffer time. To our knowledge effect of mobility model on DTN routing protocols over these metrics has not been studied using simulator. We simulate popular mobility models like random walk, random walkway point, movement based mobility, shortest path based map mobility model and working day movement model and compared the performance of routing protocols. In the next section, we present related work. In section 3 we discuss the performance metrics used for our analysis. Section 4discuss the simulation environment and section 5 analyses the results of simulation. Section 6 concludes the paper.

\section{RELATED WORK}

\section{A. Mobility Models}

Many surveys are available on the characteristics of mobility models that are used in wireless communication and most of them are also applied to delay tolerant networks. Mobility models are generally classified as: Random mobility models, Map constrained mobility models, social mobility models and composite mobility models. In this paper we have selected five most popular models for simulation and see their impact on the routing protocols of DTNs. We will see the general behaviour of these mobility models in this section.

Random Walk (RW)model mimics the natural movements of the various entities in unpredictable direction. Every node in this model moves towards a randomly chosen location. There is predefined range of speed and direction ([Speedmin,Speedmax $]$ and $[0,2 \pi]$ ) from which each node is assigned a speed and direction. After reaching to the destination a node is again assigned a new direction and speed randomly. Random walk mobility model is a memory less mobility pattern and does not stores past speed and direction. Current speed and direction of a node is independent of previous speed and direction of the node [1]. Random Walkway Point(RWP) is a mobility model which is used widely for the simulation purpose. In this mobility model a random pause time is introduced in between each movement of a node. A node takes a pause before changing its speed and direction. If pause time is taken zero then Random Walkway Point works similar to Random walk mobility model.Random Walkway point model is mathematically analysable and easy to implement [2].Using the characteristic of this model Abdulla and Simon provide some very important results in [3]: 
Probability that a message is delivered before it is expired can be given as

$$
E[R]=1-e^{-\gamma T_{x}}
$$

Where node arrival times are divided exponentially distributed with a rate of $\gamma$ and $T_{x}$ is the message expiration time. Expected waiting time for a delivered message can be given as

$$
E(T)=\frac{1}{\gamma}-\frac{e^{-\gamma T_{X}}}{1-e^{-\gamma T_{X}}} T_{X}
$$

Expected number of messages in the buffer for each node can be given as

$$
N=\frac{\lambda}{\gamma}(M-1)\left(1-e^{-\gamma T_{x}}\right)
$$

where $\lambda$ is message generation rate and there are M mobile nodes.

Map based mobility model (MBM) restricts the node movement based on map data which defines the road on the map to be followed by a node. Direction of node is determined randomly similar to random Walk model. This model restricts the movement area geographically. This model gives the option to divide the nodes in group based on the portion of map used by groups. Group of car can be given separate portion of the map from the pedestrian. To better model the real-world mobility MBM limit the node movement to predefined paths derived from real map data. Map data is defined in Well Known Text (WKT) and MBM understands this data. For simulation purpose nodes are placed randomly and are moved on a path continuously unless they touch the end of road or reach to the intersection. If end of road is reached the node returns back and if the node is on intersection, then it selects a new path other than the previous path through which it came from [2] [4]. Shortest Path Map Based Mobility model (SPMBM) is an improved version of Map based movement model.This model uses Dijkstra's shortest path algorithm for the movement of nodes on the map. Initially nodes are placed randomly on the map and move towards a destination in the map following the Dijsktra's algorithm. When a node reaches to the destination after waiting for a moment it has to select a new destination. Although all places have the equal probability to be selected as destination for better model reality some points of interests (POIs)are added in map data. Probabilities of selecting as next destination for POIs may be defined for the certain group of nodes. These POI's may be used to represent some special spot of attractions like cinema hall, Restaurant, Collegeetc [5].

Working Day Movement Model (WDM)consists of different major activities: activities at home, at work and in the evening. Home activity sub model is used for evening and night activities. Once a node reaches its home location (a point on the map) it walks a short distance away and stays still until the wakeup time. Office activity is used to model movement inside the office where an employee may move to some other colleague or toward the meeting point. Evening activities models the activities that nodes can do in evening like shopping, going to club or a bar. Transport model captures the movement of node between the home, office and evening activity. A node may walk using streets or it may use car or can use busses for travelling [6]

\section{B. Routing protocols used in DTN}

Objective of routing protocols in delay tolerant network is to increase delivery probability at its maximum and reduce the delivery delay to its minimum. Several protocols have been proposed by researchers to be used in delay tolerant network and following is brief introduction of the protocols which are very popular. In Epidemic routing protocol,no knowledge is used and maximum replication is used. When two nodes come in contact each other they first exchange the list of messages they already have. After verifying from the list, they exchange copy of the messages that they don't have [7]. Epidemic routing gives the maximum delivery probability of a message towards the destination but at the cost of increased network overhead. PRoPHET(Probabilistic ROuting Protocol using History of Encounters and Transitivity) [8] uses partial knowledge and some replication. Every node maintains a metric delivery probability for other node based on contacts made in history. Messages are forwarded to other node with better probability of delivery. MaxProp[9] ranks the stored messages based on a cost of destination. Priority is given to the packet which travels fewer hops for the same cost. Packets with higher priority transferred first on contact. Spray and wait protocol makes $\mathrm{N}$ copies of packets and sprays it to $\mathrm{N}$ relays where relays are normal nodes selected to act as relay for this packet only uses knowledge. If these relays are not the destination they will deliver the packet only on direct contact with destination. Relays do not make copies [10].

\section{PERFORMANCE METRICS}

DTN routing protocols are designed to work in environment where delays need to be tolerated, so primary requirement of such protocols is reliable delivery.Hence, delivery probability is one of the metrics which we consider for evaluating the routing protocols. Other matrices include average delivery latency, overhead ratio and average buffer time. Delivery probability is defined as the ratio of the number of messages delivered to the destination and the number of messages sent by the sender. Average delivery latency is defined as the average of 
time taken by all messages to reach from source to destination. Overhead ratio is defined as the ratio of difference between the total number of relayed messages and the total numbers of delivered messages to the total number of delivered messages. Average buffer time is defined as the average of the time a message lives inside any nodes buffer in the network.

\section{SIMULTAION}

We evaluate the effect of above described mobility models on performance of different algorithms. The simulation scenario is based on the city of Helsinki presented in Figure 1[11]. Simulation specific settings for various mobility models are shown in Table I and Table II. These settings applied to three different protocols namely Epidemic, Prophet and Binary Spray and Wait brings us with the following results presented further. The simulation is done using ONE simulator [11].

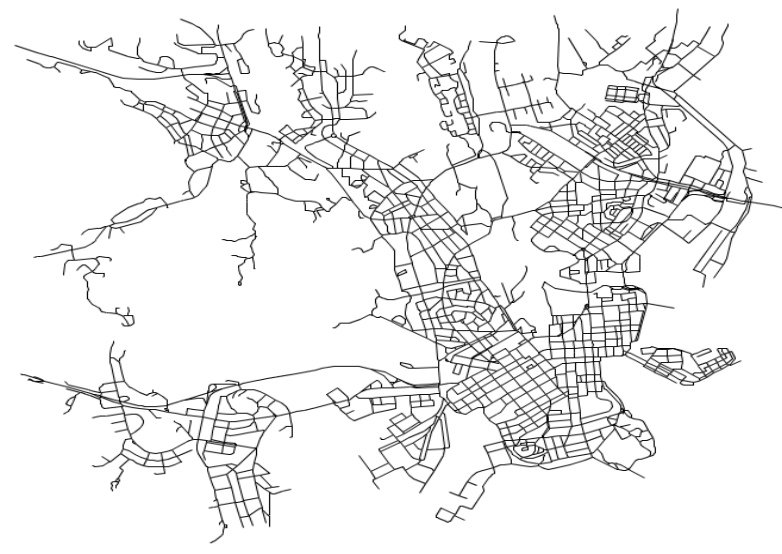

Figure 1: Helsinki simulation scenario $(10000 \times 8000 \mathrm{~m})$

Table I: Simulation Parameters for RandomWalk/ RandomWaypoint/ MapBasedMovement/ ShortestPathMapBasedMovement models

\begin{tabular}{|l|l|}
\hline Parameter & Value \\
\hline Simulation Time (hours) & 48 \\
\hline Update Interval (seconds) & 1 \\
\hline Warm-up Time (seconds) & 900 \\
\hline Bundle Time-to-Live (hours) & 5 \\
\hline Buffer Size (Mbytes) & $100 \mathrm{MB}$ \\
\hline Number of Interfaces & 1 \\
\hline Transmission Range (meters) & 10 \\
\hline Transmission Speed (kBps) & 100 \\
\hline Number of Nodes & 400 \\
\hline Number of Copies of message in SnW (Binary) & 10 \\
\hline Seconds in time out (Prophet) & 30 \\
\hline Node Movement & {$[\mathrm{RW} ; \mathrm{RWP} ;$ MBM; SPMBM;] } \\
\hline Routing Protocol & {$[$ Epidemic; Prophet; Spray and Wait] } \\
\hline Node Speed (m/s) & $0.5 \sim 5$ \\
\hline Wait Time (seconds) & $0 \sim 8$ \\
\hline Message creation interval(sec) & $15 \sim 20$ \\
\hline Message Size (Kbytes) & $500 \sim 1000$ \\
\hline Movement Random Seed & 8372 \\
\hline Map Size (meters) & $10000 \times$ 8000 \\
\hline Map File (for Map Based Movement) & Helsinki City \\
\hline
\end{tabular}


Table II: Simulation Parameters for Working Day Movement model

\begin{tabular}{|c|c|c|c|c|c|c|c|c|}
\hline \multicolumn{5}{|l|}{ Parameter } & \multicolumn{4}{|c|}{ Value } \\
\hline \multicolumn{5}{|c|}{ Simulation Time (hours) } & \multicolumn{4}{|l|}{48} \\
\hline \multicolumn{5}{|c|}{ Update Interval (seconds) } & \multicolumn{4}{|l|}{1} \\
\hline \multicolumn{5}{|c|}{ Warm-up Time (seconds) } & \multicolumn{4}{|l|}{900} \\
\hline \multicolumn{5}{|c|}{ Bundle Time-to-Live (hours) } & \multicolumn{4}{|l|}{5} \\
\hline \multicolumn{5}{|c|}{ Buffer Size (Mbytes) } & \multicolumn{4}{|c|}{$100 \mathrm{MB}$} \\
\hline \multicolumn{5}{|c|}{ Number of Interfaces } & \multicolumn{4}{|l|}{1} \\
\hline \multicolumn{5}{|c|}{ Transmission Range (meters) } & \multicolumn{4}{|l|}{10} \\
\hline \multicolumn{5}{|c|}{ Transmission Speed (kBps) } & \multicolumn{4}{|l|}{250} \\
\hline \multicolumn{5}{|c|}{ Number of Nodes } & \multicolumn{4}{|l|}{400} \\
\hline \multicolumn{5}{|c|}{ Routing Protocol } & \multicolumn{4}{|c|}{ [Epidemic; Prophet; Spray and Wait] } \\
\hline \multicolumn{5}{|c|}{ Number of Copies of message in SnW (Binary) } & \multicolumn{4}{|l|}{10} \\
\hline \multicolumn{5}{|c|}{ Seconds in time out (Prophet) } & \multicolumn{4}{|l|}{30} \\
\hline \multicolumn{5}{|c|}{ Node Speed (m/s) } & \multicolumn{4}{|c|}{$0.5 \sim 5$} \\
\hline \multicolumn{5}{|c|}{ Wait Time (seconds) } & \multicolumn{4}{|l|}{$0 \sim 8$} \\
\hline \multicolumn{5}{|c|}{$\begin{array}{l}\text { Message creation interval } \\
\text { (sec) }\end{array}$} & $15 \sim 2$ & & & \\
\hline Message Size & es) & & & & $500^{\sim}$ & & & \\
\hline Movement Ra & $\mathrm{n} \mathrm{Se}$ & & & & 8372 & & & \\
\hline Map Size (me & & & & & 1000 & 00 & & \\
\hline Map File (for I & Base & vem & & & $\begin{array}{l}\text { Helsir } \\
\text { (data }\end{array}$ & ty & $\mathrm{m} / \mathrm{rc}$ & $v k t)$ \\
\hline Settings for e & tion & orkir & $\mathbf{M}$ & & & & & \\
\hline Woking Day N & nen & & & & & & & \\
\hline GroupID & A & B & C & D & $E$ & $\mathbf{F}$ & G & $\mathbf{H}$ \\
\hline $\begin{array}{l}\text { Number of } \\
\text { Nodes }\end{array}$ & 50 & 20 & 30 & 30 & 30 & 50 & 50 & 73 \\
\hline $\begin{array}{l}\text { busControlS- } \\
\text { ystem } \mathrm{Nr}\end{array}$ & 1 & 2 & 3 & 4 & 5 & 6 & 7 & 8 \\
\hline Map File & & & & & & & & \\
\hline Meeting Spot & & & & & $\begin{array}{l}/ \text { dat } \\
<\mathrm{Gr}\end{array}$ & $\begin{array}{l}\text { Isinki } \\
>\text { Im }\end{array}$ & $\begin{array}{l}\text { um/ } \\
\text { gspo }\end{array}$ & \\
\hline
\end{tabular}




\begin{tabular}{|c|c|c|c|c|c|c|c|c|}
\hline \multicolumn{5}{|c|}{ Office Location File } & \multicolumn{4}{|c|}{$\begin{array}{l}\text { /data/HelsinkiMedium/- } \\
\text { <GroupID>_offices.wkt }\end{array}$} \\
\hline \multicolumn{5}{|c|}{ Home Location File } & \multicolumn{4}{|c|}{$\begin{array}{l}\text { /data/HelsinkiMedium/- } \\
\text { <GroupID>_homes.wkt }\end{array}$} \\
\hline \multicolumn{5}{|c|}{ Node Speed $(\mathrm{m} / \mathrm{s})$} & \multicolumn{4}{|c|}{$0.8 \sim 1.4$} \\
\hline \multicolumn{5}{|c|}{ Node Wait Time (seconds) } & \multicolumn{4}{|l|}{0} \\
\hline \multicolumn{5}{|c|}{ Probability to Own Car } & \multicolumn{4}{|c|}{0.5} \\
\hline \multicolumn{9}{|c|}{ Bus Movement: } \\
\hline GroupID & o & $\mathbf{p}$ & q & $r$ & s & $\mathbf{t}$ & $\mathbf{u}$ & $\mathbf{v}$ \\
\hline $\begin{array}{l}\text { Number of } \\
\text { Nodes }\end{array}$ & 2 & 2 & 2 & 2 & 2 & 2 & 2 & 4 \\
\hline $\begin{array}{l}\text { busControls- } \\
\text { ystem Nr }\end{array}$ & 1 & 2 & 3 & 4 & 5 & 6 & 7 & 8 \\
\hline \multicolumn{5}{|l|}{ Route File } & \multicolumn{4}{|c|}{$\begin{array}{l}\text { data/HelsinkiMedium/- } \\
\text { <GrouplD>_bus.wkt }\end{array}$} \\
\hline \multicolumn{5}{|c|}{ Bus Movement Node Speed (m/s) } & \multicolumn{4}{|c|}{ 7 10 } \\
\hline \multicolumn{5}{|c|}{ Bus Movement Nodes Wait Time (seconds) } & \multicolumn{4}{|c|}{$10 \sim 30$} \\
\hline \multicolumn{5}{|c|}{ Bus Movement Nodes Route Type } & \multicolumn{4}{|c|}{ Pingpong } \\
\hline \multicolumn{9}{|c|}{ Shortest Path MapBasedMovement: } \\
\hline \multicolumn{5}{|l|}{ GroupID } & \multicolumn{4}{|l|}{ K } \\
\hline \multicolumn{5}{|c|}{ Number of Nodes } & \multicolumn{4}{|c|}{50} \\
\hline \multicolumn{5}{|c|}{ Wait Time (seconds) } & \multicolumn{3}{|c|}{$100 \sim 300$} & \\
\hline \multicolumn{5}{|c|}{ Node Speed $(\mathrm{m} / \mathrm{s})$} & \multicolumn{4}{|c|}{ 7 10 } \\
\hline \multicolumn{5}{|c|}{ Number of offices } & \multicolumn{4}{|c|}{50} \\
\hline \multicolumn{5}{|c|}{ Work Day Length (hours) } & 8 & & & \\
\hline Probability to & r St & $\lg a$ & ork & & 0.5 & & & \\
\hline Number of $\mathrm{Mc}$ & $\mathrm{g} \mathrm{Sp}$ & & & & 10 & & & \\
\hline Office $\min W a$ & nel & ds) & & & & & & \\
\hline Office Wait Ti & & ffic & & & 0.5 & & & \\
\hline Group Size for & ing & & & & $1 \sim 3$ & & & \\
\hline Time Diff STD & & & & & 720 & & & \\
\hline After Shoppin & & nour & & & $1 \sim 2$ & & & \\
\hline
\end{tabular}


For evaluating the effect of different mobility models on the routing protocols discussed above we have taken the various transmission range i.e. 10, 50, 100, 150 and 250 meters. We studied the effect of the same with varying Bundle Time-To-Live of 30,60, 120, 300 and 600 minutes on these routing protocols. We also investigated the effect of mobility models on the routing protocols with varying number of nodes i.e. 100, 200, 400 and 800 nodes.

\section{ANALYSIS AND RESULTS}

A.Performance Analysis on basis of varying Transmission Range:

The evaluation study starts with the comparison of considered mobility models with varying transmission range of 10, 50, 100, 150 and 250 meters for Epidemic, Prophet and Spray and Wait Protocol (Figure 2-5)based on delivery probability, overhead ratio, average latency and average buffer time. As the transmission range increases, the contact duration increases and time of communication increase and combined with the movement behaviour the performance will differ.
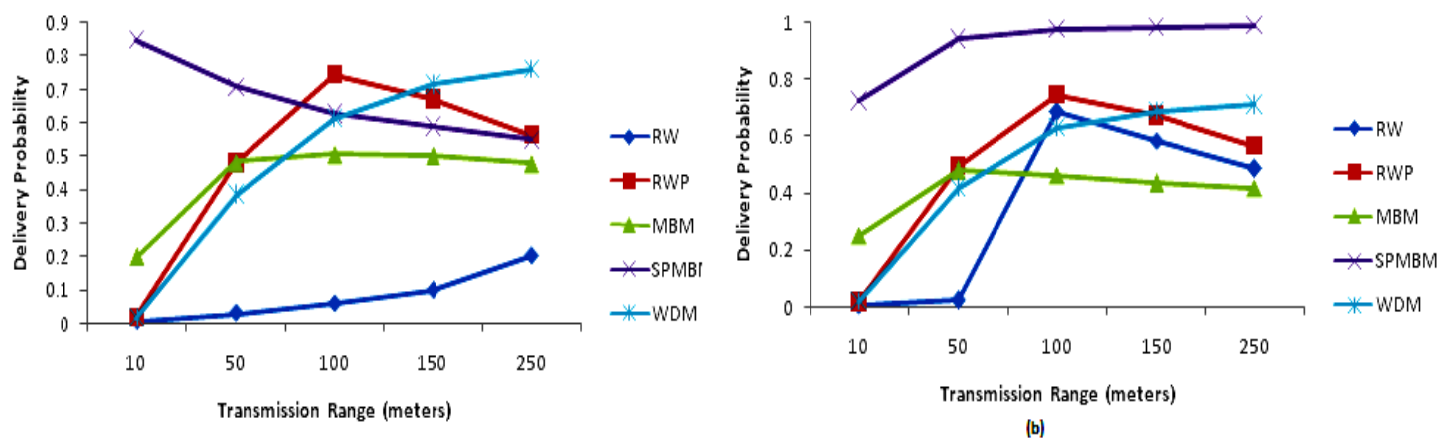

(a)

(b)

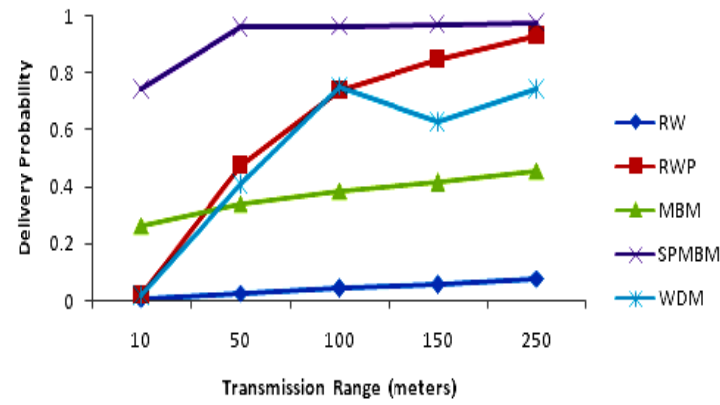

(c)

Figure 2: Delivery Probability of considered mobility models with varying transmission range for a)Epidemic b) Prophet c)Spray and Wait Routing protocol

Delivery Probability of RW model is low in case of Spray and Wait and Epidemic protocol (Figure 2) whereas when probabilistic protocol PRoPHET is used the delivery probability increases comparatively. Although it decreases for more than $100 \mathrm{~m}$ range but still more than other protocols. In case of RWP, the delivery probability curve is almost same for all three except for Spray and Wait where it goes on increasing and achieves to almost 0.93 . Similar characteristics are shown by MBM model but the delivery probability is not that high compared to RWP, WDM and SPMBM. Since SPMBM uses shortest path towards the destination using Dijikstra's algorithm, SPMBM model outperforms all the models and achieves a high delivery probability except in case of pure flooding based protocol (Epidemic). 

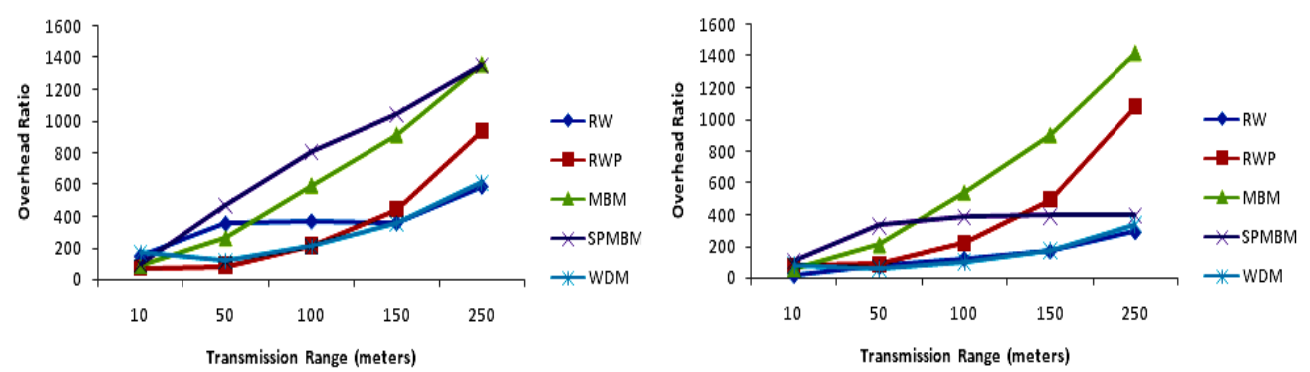

(a)

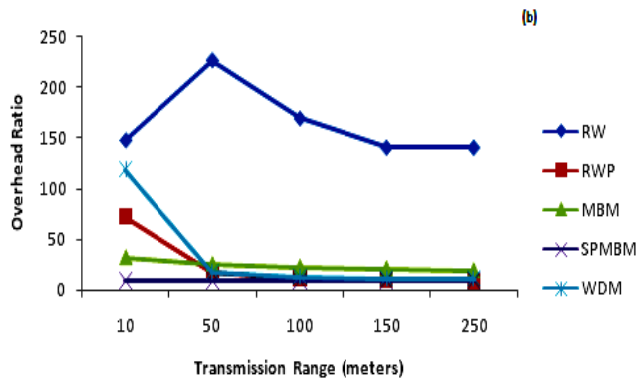

(c)

Figure 3: Overhead Ratio of considered mobility models with varying transmission range for a) Epidemic b) Prophet c )Spray and Wait Routing protocol

It can be seen from the figure 3overhead ratios increases with the increase in transmission range. This is due to the fact that as transmission range increases there is more replication of messages are there and so this effect is same in all mobility models. MBM models have more overhead because of restricted movement to map area. The exception is the Spray and Wait protocol which has lower overhead because of restricted number of copies.

More transmission range gives opportunity for nodes to transfer messages from a distance and so a mobile node not very nearby to a destination node can deliver the bundles easily and so the average latency decreases with increase in transmission range. We see that SPMBM has lowest latency this is because it proceeds towards the destination by selecting the shortest path available and so bundles will be delivered faster (Figure 4).

We see average buffer time decreases (Figure 5) with increase in transmission range as more bundles are relayed due to flooding nature of the algorithms and so in Epidemic and Prophet bundles will be dropped once the buffer becomes full. In case of spray and wait we see the graphs are similar, this is due to the fact that number of copies are restricted and so replication is controlled. In all three protocols, there is no scheme of acknowledgement and so bundle will live in the buffer till it is dropped.

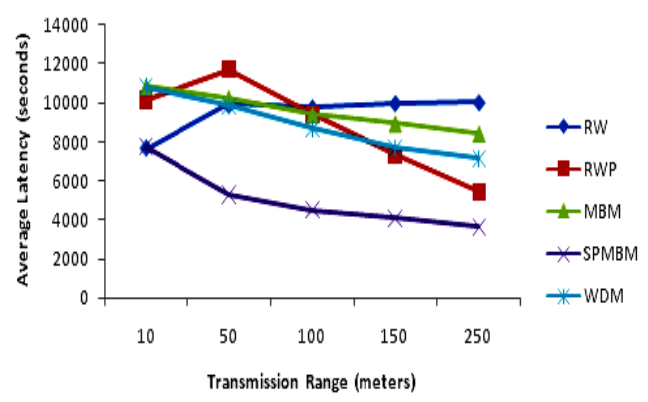

(a)

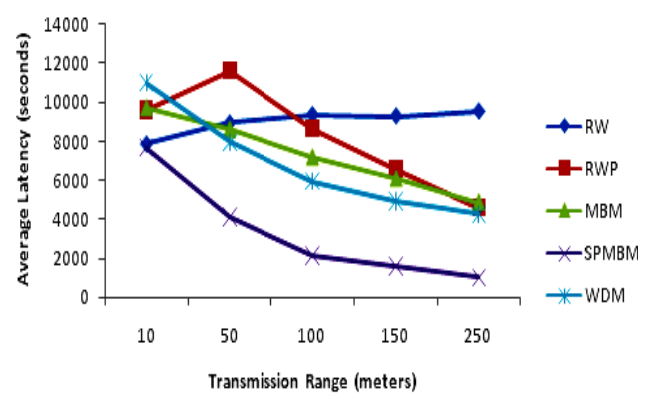

(b)

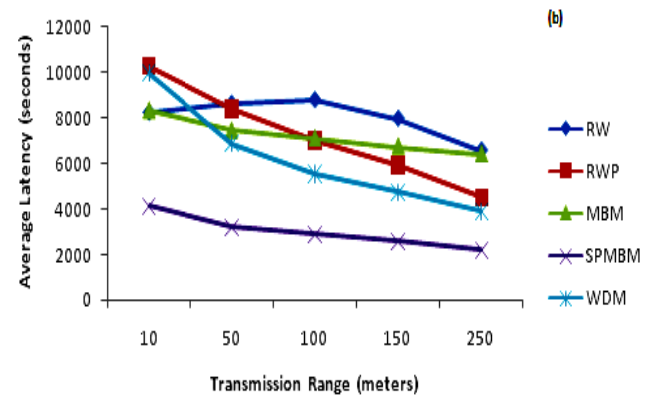

(c)

Figure 4: Average Latency of considered mobility models with varying transmission range for Routing protocol

a) Epidemic b) Prophet c) Spray and Wait 


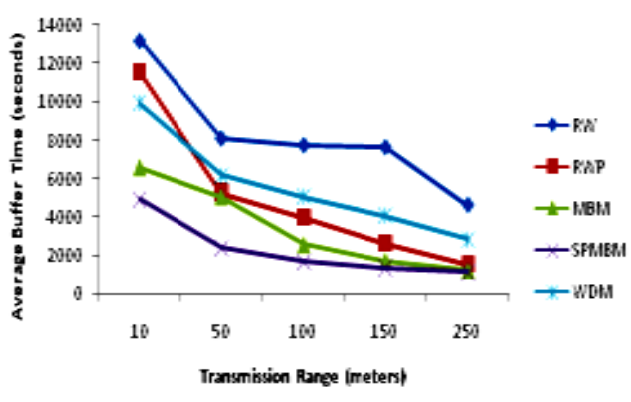

(a)

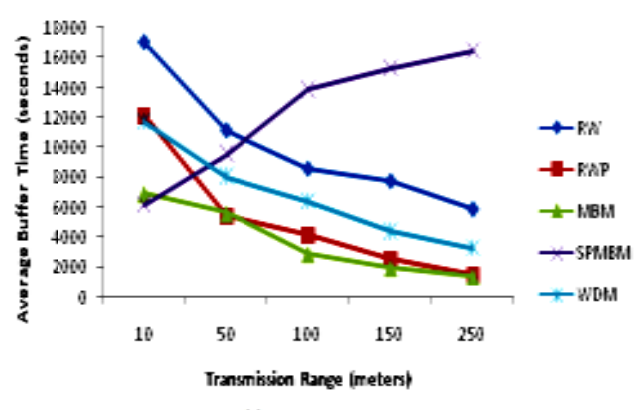

a)

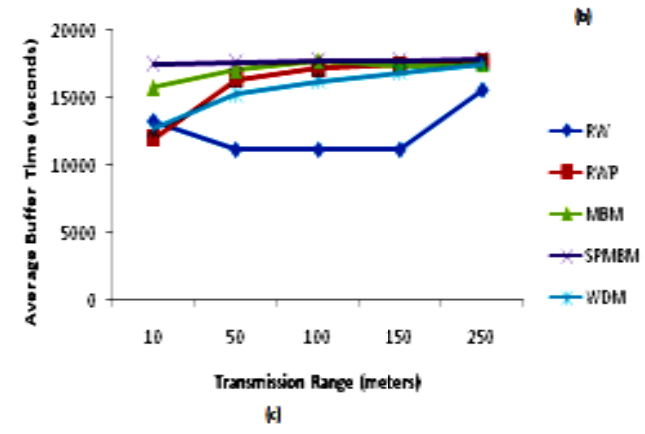

Figure 5: Average Buffer Time of considered mobility models with varying transmission range for a)Epidemic b)Prophet c)Spray and Wait Routing protocol

\section{B. Performance Analysis on basis of varying Bundle Time-To-Live}

Next, we evaluate our considered mobility models with varying Bundle Time-To-Live of 30, 60, 120, 300 and 600 minutes for Epidemic, Prophet and Spray and Wait Protocol (Figure 6-9) based on delivery probability, overhead ratio, average latency and average buffer time. As the Time-To-Live increases, the bundles get more time to survive in network and combined with the movement behaviour changes occur in inter contact time and nature of unique encounter and this may affect the performance of the network.
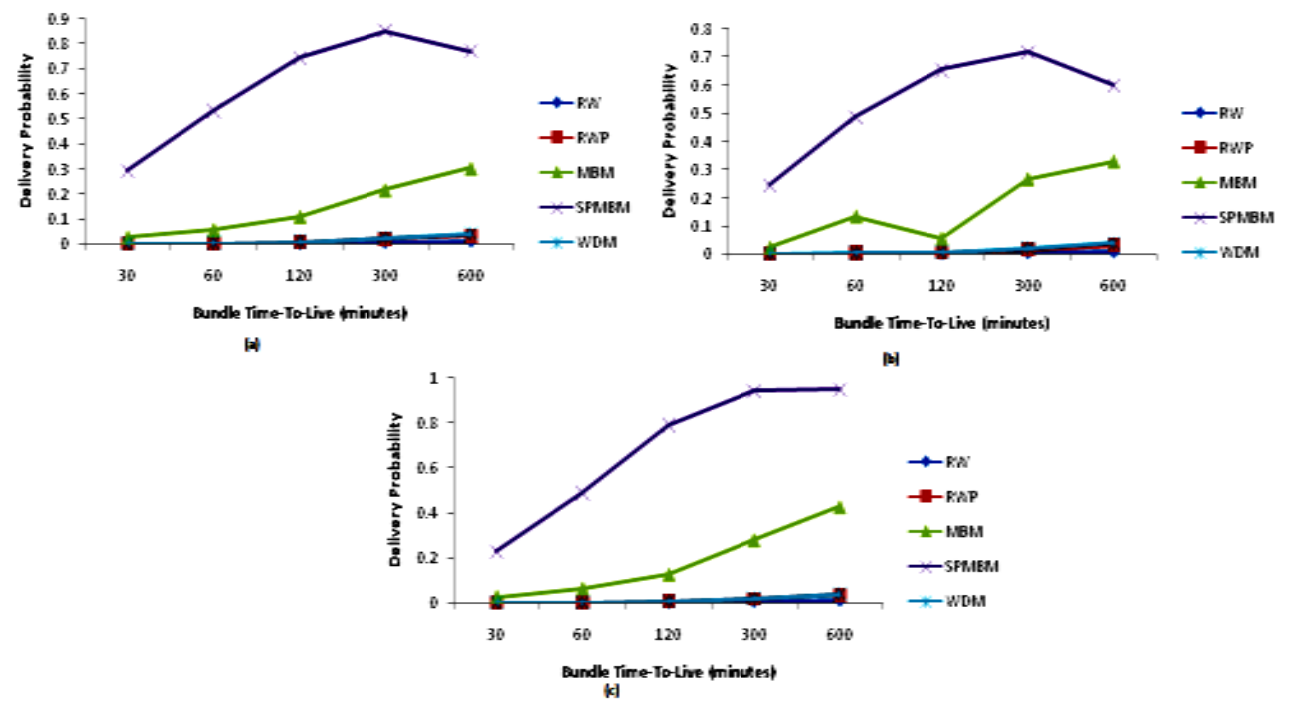

Figure 6: Delivery Probability of considered mobility models with varying bundle time-to-live fora) Epidemicb)Prophet c) Spray and Wait Routing protocol

When we make the nodes, resource constrained with a small transmission range of $10 \mathrm{~m}$ and buffer of $100 \mathrm{M}$ we see that delivery of bundles drops drastically. The nature of SPMBM to follow the shortest path towards the destination to deliver bundles gives it advantage over other models to have a good delivery probability. Also, the delivery probability increases with use of restricted copy spray and wait protocol which allows for less bundles to be dropped as bundles will be limited in the network. MBM also takes the advantage of having restricted moving space and continuous movement of nodes and has delivery probability more than the rest of the models. In case of WDM, RW and RWP all show almost the same performance and WDM among them has performed better whereas RW has least delivery probability (Figure 6). 

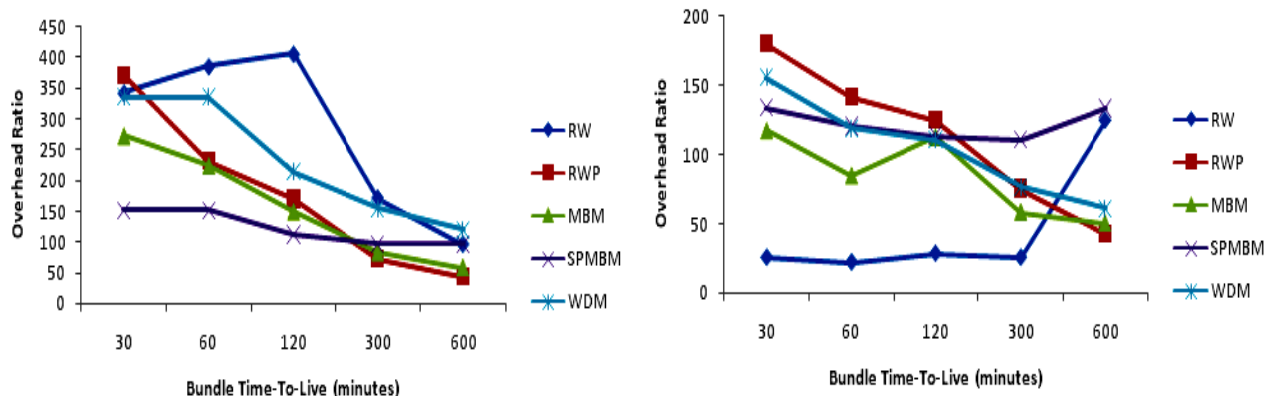

(a)

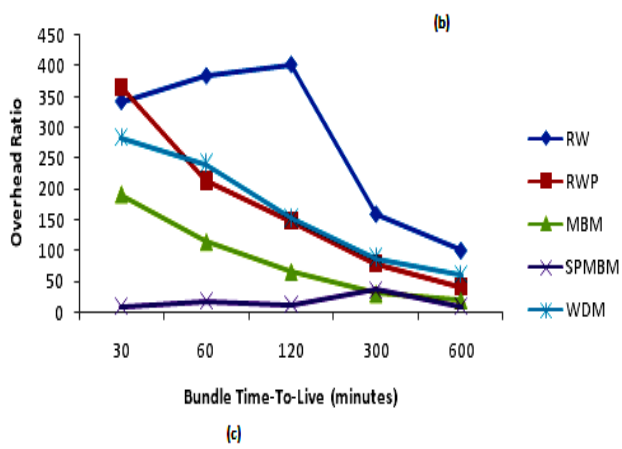

Figure 7: Overhead Ratio of considered mobility models with varying bundle time-to-live for a) Epidemic b) Prophet c)Spray and Wait Routing protocol

Overhead ratio has similar behaviour for all the movement models with varying TTL. With increase in TTL the overhead ratio decreases and SPMBM giving the least overhead ratio in case of Epidemic and spray and wait. Increasing the TTL gives impact to decrease the overhead ratio so, as a result it is better for network if have higher time to live (Figure 7).

Average latency of bundles in any mobility model shoots up as we increase the TTL. From Figure 8 we have seen that the delivery probability is very less and bundles have not been delivered, with increase in TTL value bundles stayed more time in the buffer but were not delivered and even if delivered was after a long time. With the use of intelligent probabilistic protocol (Prophet) the latency is reduced for SPMBM, MBM and RW as compared to epidemic. And spray and wait gives advantage to SPMBM and MBM more than prophet.

Increase in message TTL gives a bundle more chance to reside in buffer and so as TTL increases average buffer time also increases. The application of probabilistic based forwarding does not have any effect on bundle's time to live in buffer. Here, SPMBM and MBM have an edge over other mobility models with WDM being just next to them (Figure 9)

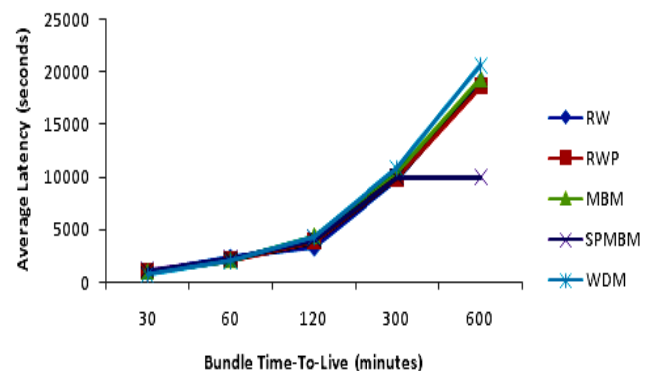

Bundle Time-To-Live (minutes)

(a)

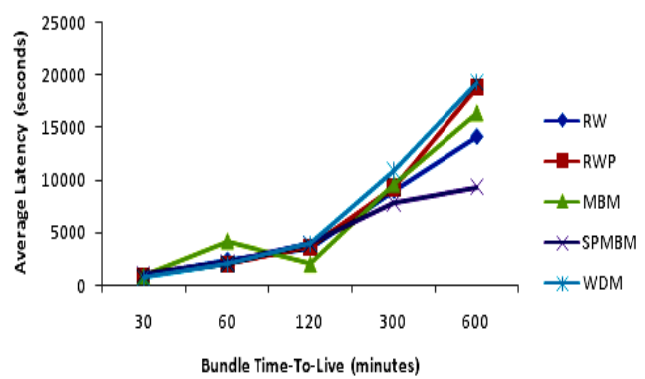

(b)

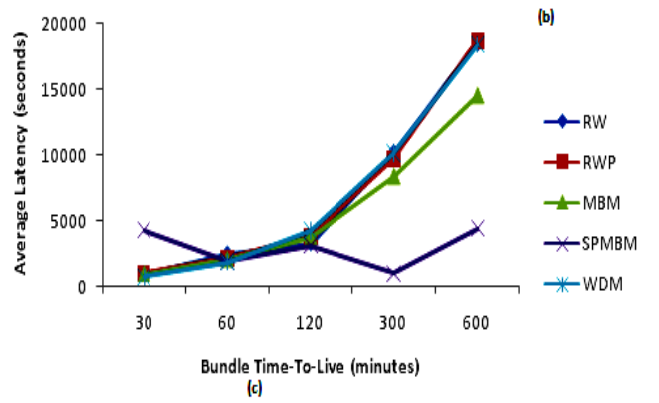

(c)

Figure 8: Average Latency of considered mobility models with varying bundle time-to-live fora) Epidemic b) Prophet c) Spray And Wait Routing protocol 


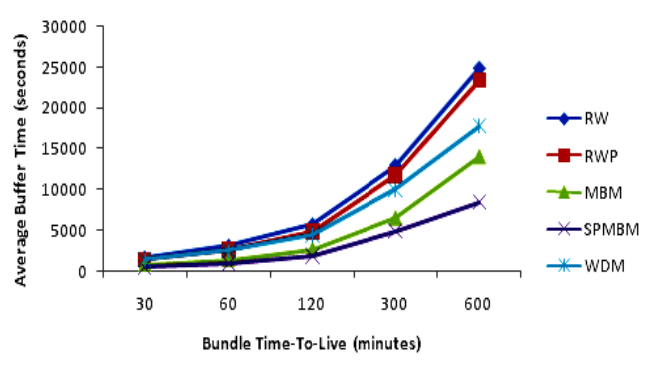

(a)
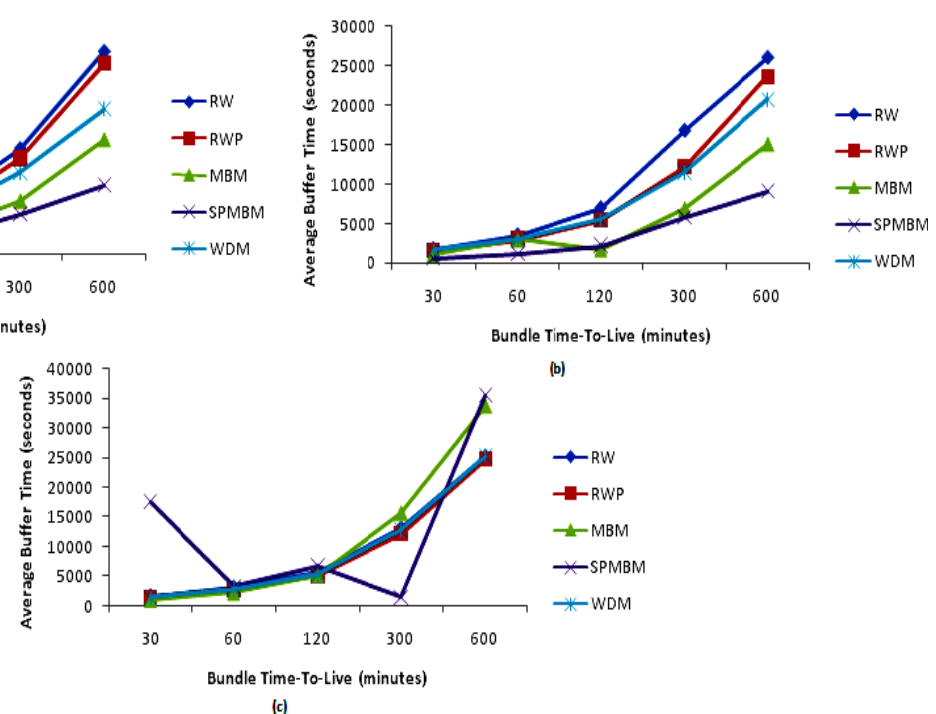

(c)

Figure 9: Average Buffer Time of considered mobility models with varying bundle time-to-live for a)Epidemic b) Prophet c) Spray And Wait Routing protocol

\section{C.Performance Analysis on basis of varying Number of Nodes}

Further, we evaluate our considered mobility models with varying number of nodes of 100, 200, 400 and 800 for Epidemic, Prophet and Spray and Wait Protocol (Figure 10-14) on the basis of delivery probability, overhead ratio, average latency and average buffer time. As the Number of Nodes increase, the inter contact time and number of encounters will differ and when mobile nodes will have different movement behaviour contact patterns will be different this may affect the performance of the network.

We have constrained the resources for the nodes and upon increasing the number of nodes also, we find that only SPMBM shows good performance, rest all of the mobility models have the performance as in case of bundle Time to Live(Figure 10).

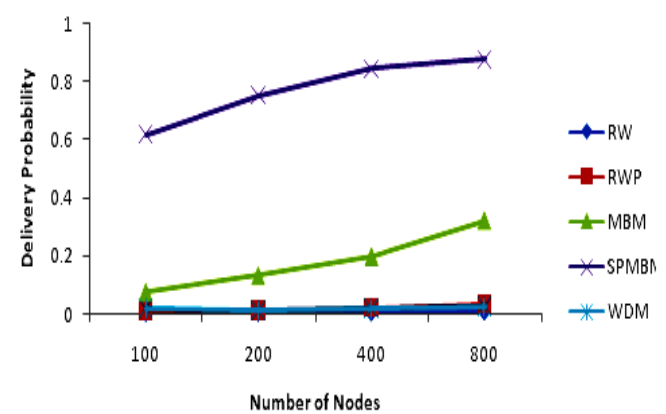

(a)

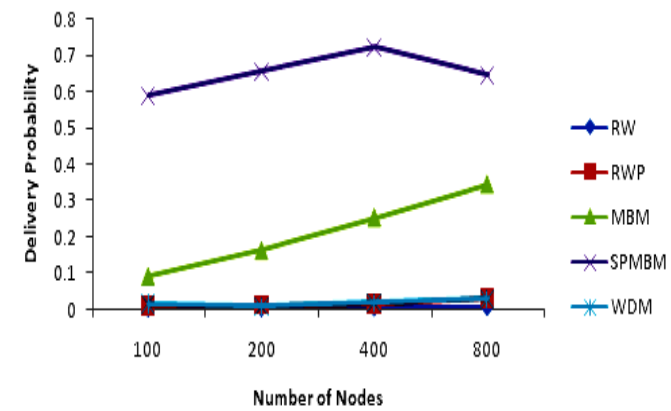

(b)

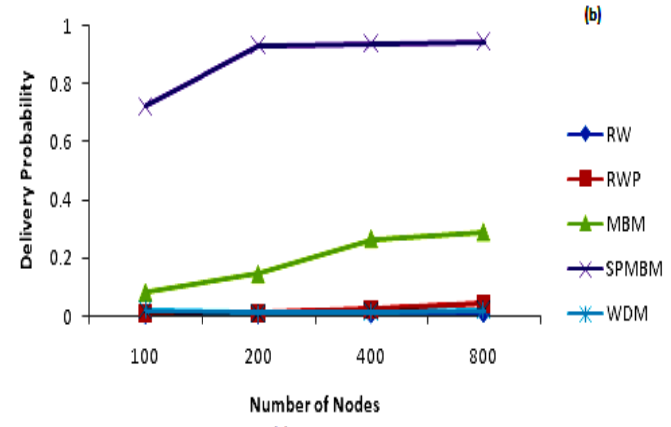

(c)

Figure 10: Delivery Probability of considered mobility models with varying number of nodes fora) Epidemic b)Prophet c) Spray And Wait protocol

The characteristic of overhead ratio here mainly depended on the nature of algorithm used. The overhead ratio is more for flooding based protocols (Epidemic and Prophet) and is low for Spray and wait(Figure 11). 

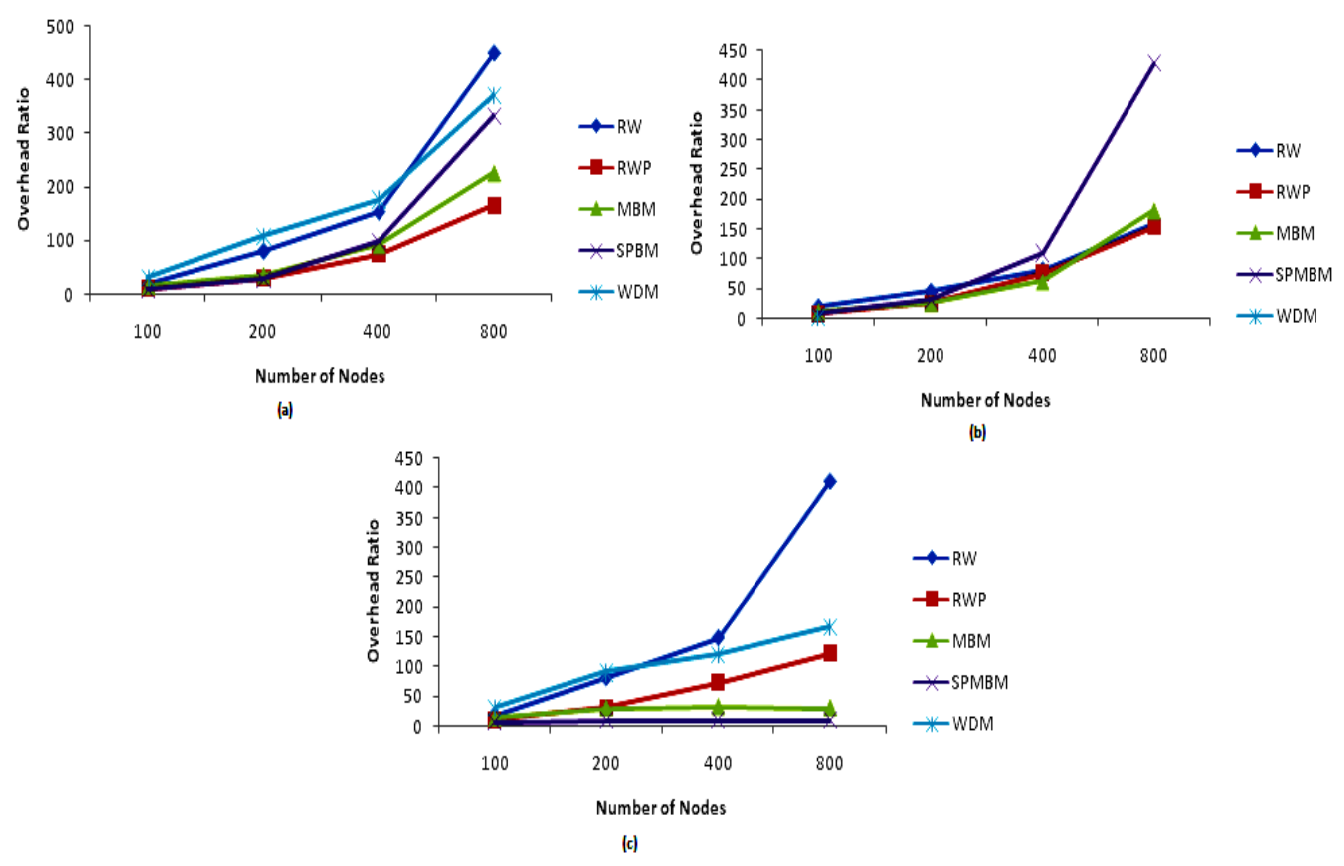

Figure 11: Overhead Ratio of considered mobility models with varying number of nodes for a) Epidemic b) Prophet c) Spray and Wait Routing protocol

Average latency is least in SPMBM and decreases with increase in number of nodes. All other mobility models show the same behaviour and show an increasing latency for nodes more than 200(Figure 12).

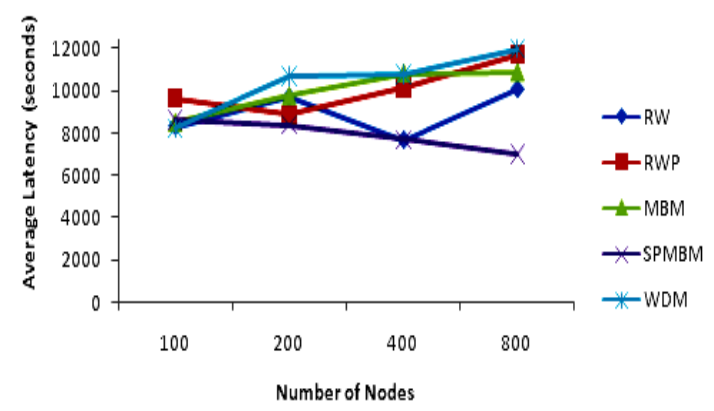

(a)

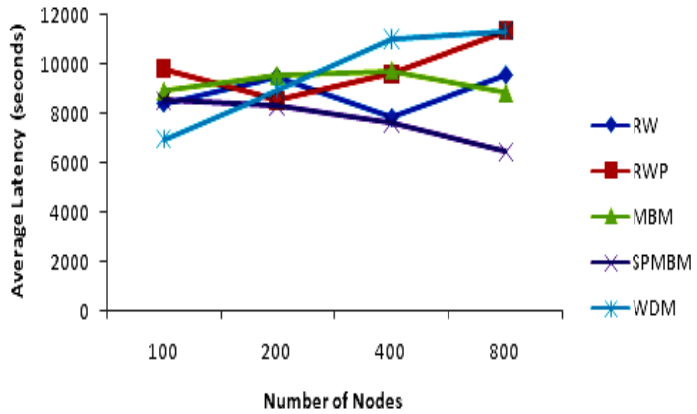

(b)

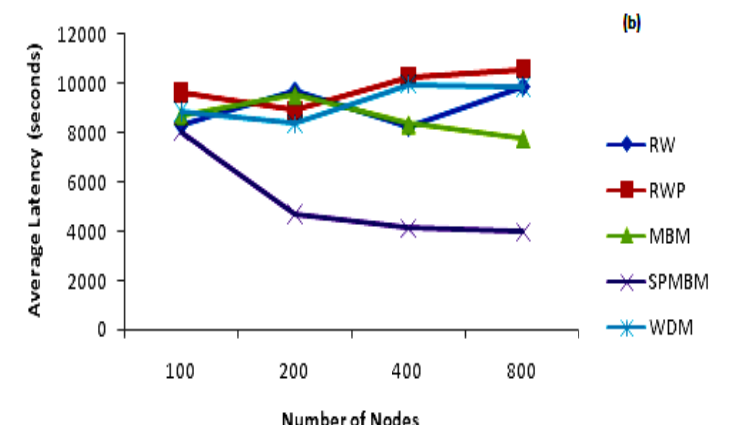

(c)

Figure 12: Average Latency of considered mobility models with varying number of nodes for a) Epidemic b) Prophet c) Spray And Wait protocol

Average buffer time decreases as number of nodes increase. With increase in nodes, more contact opportunities are registered and so are message exchanges. With a limited buffer, flooding based protocols suffer with dropping of bundles. Epidemic and Prophet show the same trend which can be verified from the graphs. Coming to Spray and Wait which is a controlled flooding scheme, limited replication of bundles gives them opportunity to stay in buffer and so with increase in number of nodes there is not much decrease in bundle buffer time( Figure 13). 

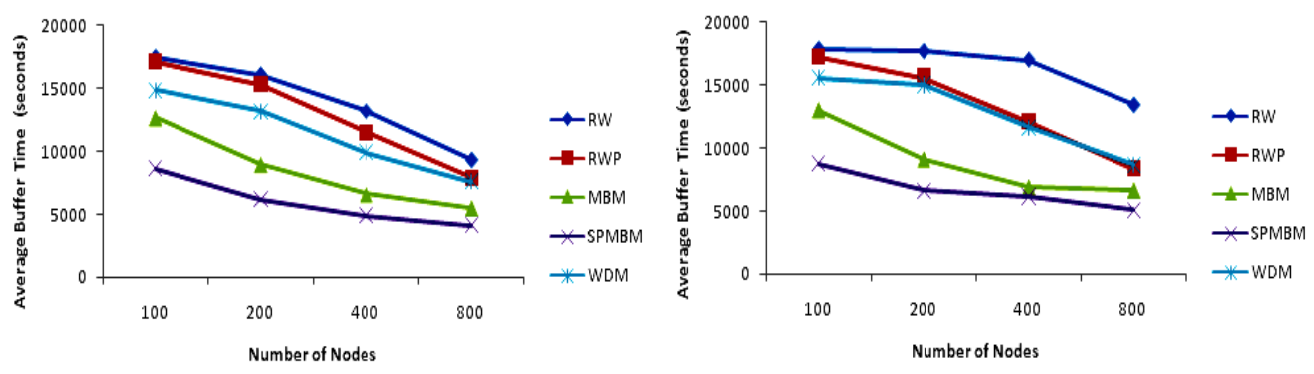

(a)

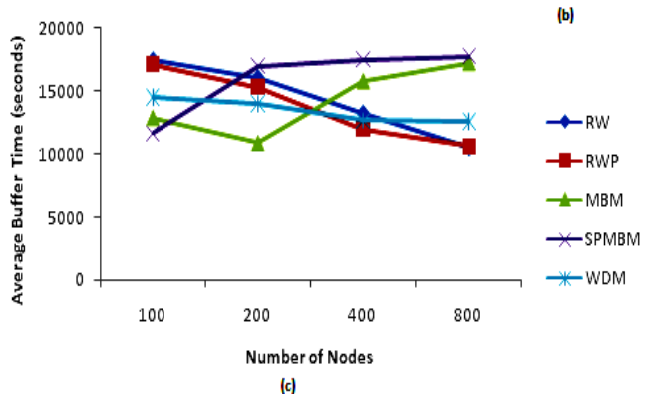

(c)

Figure 13: Average Buffer Time of considered mobility models with varying number of nodes for Spray And Wait protocol

\section{CONCLUSIONS}

In this paper we discussed various mobility models and their impact on routing protocols of delay tolerant networks. We used the ONE simulator to show the performance of three routing protocols using different mobility models under different conditions like varying the transmission rage, bundle time to live (TTL) and number nodes. We have shown the importance of mobility behaviour of nodes by simulating them while evaluating the performance of routing protocols for DTN. We explained the performance of Epidemic, PRoPHET, and SnW routing protocols on the basis of results came out from the simulation when they work with different mobility models. SPMBM mobility model gives better result in most of the cases. In future, we would like to expand our work for the more recent routing protocols developed by various researchers for DTN environment.

\section{REFERENCES}

[1] Camp, Tracy, Jeff Boleng, and Vanessa Davies. "A survey of mobility models for ad hoc network research." Wireless communications and mobile computing 2.5 (2002): 483-502.

[2] Shahzamal, M., et al. "Mobility Models For Delay Tolerant Network: ASurvey." International Journal of Wireless \& Mobile Networks 6.4 (2014).

[3] Abdulla, Muhammad, and Robert Simon. "The impact of the mobility model on delay tolerant networking performance analysis." Simulation Symposium, 2007. ANSS'07. 40th Annual. IEEE, 2007.

[4] Keränen, Ari, Jörg Ott, and Teemu Kärkkäinen. "The ONE simulator for DTN protocol evaluation." Proceedings of the 2nd international conference on simulation tools and techniques. ICST (Institute for Computer Sciences, Social-Informatics and Telecommunications Engineering), 2009.

[5] Keränen, Ari, and Jörg Ott. "Increasing reality for dtn protocol simulations." Helsinki University of Technology, Tech. Rep (2007).

[6] Ekman, Frans, et al. "Working day movement model." Proceedings of the 1st ACM SIGMOBILE workshop on Mobility models. ACM, 2008.

[7] Vahdat, Amin, and David Becker. Epidemic routing for partially connected ad hoc networks. Technical Report CS-200006, Duke University, 2000

[8] Lindgren, Anders, Avri Doria, and Olov Schelen. "Probabilistic routing in intermittently connected networks." Service Assurance with Partial and Intermittent Resources. Springer Berlin Heidelberg, 2004. 239-254.

[9] Burgess, John, et al. "MaxProp: Routing for Vehicle-Based Disruption-Tolerant Networks." INFOCOM. Vol. 6. 2006

[10] Spyropoulos, Thrasyvoulos, Konstantinos Psounis, and Cauligi S. Raghavendra. "Spray and wait: an efficient routing scheme for intermittently connected mobile networks." Proceedings of the 2005 ACM SIGCOMM workshop on Delay-tolerant networking. ACM, 2005.

[11] Keränen, Ari, Jörg Ott, and Teemu Kärkkäinen. "The ONE simulator for DTN protocol evaluation." Proceedings of the 2nd international conference on simulation tools and techniques. ICST (Institute for Computer Sciences, Social-Informatics and Telecommunications Engineering), 2009.

[12] Fall, Kevin. "A delay-tolerant network architecture for challenged internets." Proceedings of the 2003 conference on Applications, technologies, architectures, and protocols for computer communications. ACM, 2003. 


\section{Author Profile}

Sanjay Kumar is working as Assistant Professor in National Institute of Technology, Raipur (India).He received M.Tech.(CSE) in 2007 from RGPV Bhopal, India. His research focuses on wireless ad-hoc networks and Delay Tolerant Networks.

Sudhakar Pandey received D.Phil. from University of Allahabad, India in 2012.He is currently working as Assistant Professor in NIT Raipur, India. His research focuses on wireless networks, Delay Tolerant Networks and Wireless sensor networks. 